\title{
A Signal-Dependent Time-Frequency Representation: Fast Algorithm for Optimal Kernel Design
}

\author{
Richard G. Baraniuk, Member, IEEE, and Douglas L. Jones, Member, IEEE
}

\begin{abstract}
A time-frequency representation based on an optimal, signal-dependent kernel has been proposed recently in an attempt to overcome one of the primary limitations of bilinear time-frequency distributions: that the best kernel and distribution depend on the signal to be analyzed. The optimization formulation for the signal-dependent kernel results in a linear program with a unique feature: a tree structure that summarizes a set of constraints on the kernel. In this paper, we present a fast algorithm based on sorting to solve a special class of linear programs that includes the problem of interest. For a kernel with $Q$ variables, the running time of the algorithm is $O(Q \log Q)$, which is several orders of magnitude less than any other known method for solving this class of linear programs. This efficiency enables the computation of the signal-dependent, optimal-kernel time-frequency representation at a cost that is on the same order as a fixed-kernel distribution. An important property of the optimal kernel is that it takes on essentially only the values of 1 and 0.
\end{abstract}

\section{INTRODUCTION}

$\mathbf{T}$ THE BILINEAR time-frequency distributions (TFD's) of Cohen's class [1]-[3] have proven effective for analyzing the time-varying frequency content of nonstationary signals. A TFD $P(t, \omega)$ from Cohen's class measures the energy content of a signal as a function of both time $t$ and frequency $\omega$, and can be expressed as

$$
P(t, \omega)=\frac{1}{2 \pi} \int_{-\infty}^{\infty} \int_{-\infty}^{\infty} A(\theta, \tau) \Phi(\theta, \tau) e^{-j \theta_{t}-j \tau \omega} d \theta d \tau
$$

where $A(\theta, \tau)$ is the symmetrical ambiguity function (AF) of the signal to be analyzed, and $\Phi(\theta, \tau)$ is a function called the kernel of the TFD. In practice (for discrete-time signals), TFD's are computed at discrete time and frequency points; discretization of (1) yields a formula for computing a discrete TFD as the two-dimensional fast Fourier transform (FFT) of the product of a discrete $\mathrm{AF} A_{d}(m, n)$ and a discrete kernel $\Phi_{d}(m, n)$.

Manuscript received December 24, 1991; revised August 19, 1992. The associate editor coordinating the review of this paper and approving it for publication was Prof. Georgios B. Giannakis. This work was supported by the Sound Group of the Computer-based Education Research Laboratory, University of Illinois, the Joint Services Electronics Program under Grant N00014-90-J-1270, and the National Science Foundation under Grant MIP90-12747.

R. G. Baraniuk is with the Department of Electrical and Computer Engineering, Rice University, Houston, TX 77251-1992.

D. L. Jones is with the Department of Electrical and Computer Engineering, University of Illinois, Urbana, IL 61801.

IEEE Log Number 9213274
The free choice of the kernel function in (1) gives rise to the considerable flexibility of the bilinear TFD's for signal analysis purposes. Each choice of a different kernel function $\Phi(\theta, \tau)$ yields a different TFD $P(t, \omega)$, and hence a different representation of the time-frequency content of the signal. Examples of bilinear TFD's employing fixed kernels include the spectrogram (the squared magnitude of the short-time Fourier transform), the Wigner and smoothed Wigner distributions, the Choi-Williams distribution [4], and the cone-kernel distribution [5]. However, as demonstrated in a companion paper [6], a TFD based on a single, fixed kernel will provide an accurate time-frequency representation only for a limited class of signals.

In an attempt to mitigate this fundamental limitation of fixed-kernel TFD's, we have proposed in [6] a new TFD based on a signal-dependent kernel that automatically changes with each signal to ensure an accurate time-frequency representation for a large class of signals. To find the "best" kernel for each individual signal, the optimal discrete kernel $\Phi_{d}^{\text {opt }}(m, n)$ for a signal is defined as the solution to the following optimization problem-a special linear program in variables $\left|\Phi_{d}(m, n)\right|^{2}$ :

Linear Program 1 (LPl)

$$
\max _{\Phi_{d}} \sum_{m=-(M / 2)}^{M / 2-1} \sum_{n=-(N / 2)}^{N / 2-1}\left|A_{d}(m, n)\right|^{2}\left|\Phi_{d}(m, n)\right|^{2}
$$

subject to

$$
\Phi_{d}(0,0)=1
$$

$$
\Phi_{d}(m, n) \text { is radially nonincreasing }
$$

$$
\frac{\Delta_{\theta} \Delta_{\tau}}{2 \pi} \sum_{m=-(M / 2)}^{M / 2-1} \sum_{n=-(N / 2)}^{N / 2-1}\left|\Phi_{d}(m, n)\right|^{2} \leq \alpha, \quad \alpha \geq 0 .
$$

Here, $A_{d}(m, n)$ is the $M \times N$ discrete AF of the signal to be analyzed, $\alpha$ is the total kernel volume, and $\Delta_{\theta}$ and $\Delta_{\tau}$ are constants. Typically, we have $M=N=L$, with $L$ being the number of discrete signal samples to analyze. Both the $\mathrm{AF}$ and optimal kernel are computed on a rectangle grid of samples $(m, n)$. The radially nonincreasing constraint (4) is 


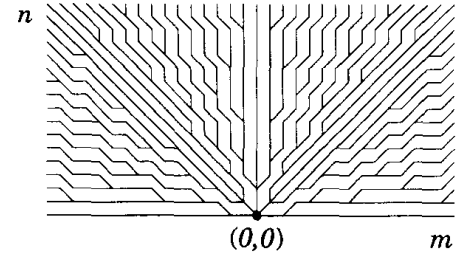

Fig. 1. A tree structure approximating a set of radial paths for the upper half-plane of a $32 \times 32$ rectangular sampling grid. The kernel nonincreasing constraint (4), (6) is implemented by forcing the kernel to be nonincreasing along branches of the tree. Details regarding construction of this tree and other trees having approximately radial branches can be found in [6].

implemented by forcing the kernel values to be nonincreasing along the branches of a special tree structure whose branches approximate a set of radial paths on the rectangular grid (see Fig. 1). More explicitly, (4) is implemented using the set of constraints

$$
\Phi_{d}(m, n) \leq \Phi_{d}[p(m, n)]
$$

where $p(m, n)$ represents the first sample point encountered when moving along the branch of the tree from sample $(m, n)$ towards the orgin $(0,0)$. See [6] for further details on the derivation of LP1.

The optimal signal-dependent TFD is obtained by computing $A_{d}(m, n)$, solving $\mathrm{LP}^{1}$ for $\Phi_{d}^{\mathrm{opt}}(m, n)$, and then taking the 2-D FFT of the product $A_{d}(m, n) \Phi_{d}^{\text {opt }}(m, n)$.

The time-frequency representation of the optimal-kernel (OK) TFD is excellent for a large class of signals [6]. However, an efficient means of computing this representation must be developed if it is to be utilized in real applications. Excepting the computation of the optimal kernel, the time to compute the OK TFD is on the same order as a fixed-kernel distribution, that is, $O\left(L^{2} \log L\right)$. Unfortunately, the costs associated with existing algorithms for solving linear programs such as LP1 are much higher, with the result that the optimal kernel solution dominates the overall cost of computing the OK TFD. For example, the worst-case time complexity of the currently most efficient algorithm for solving general linear programs is $O\left(L^{5} b\right)$, where $b \geq 1$ represents the number of digits of accuracy required in the solution [7]. Other algorithms for solving simpler "two-variables-per-constraint" linear programs could be used to solve LP1 but are no faster; the worst-case time complexity of the most efficient algorithm is $O\left(L^{8} \log L\right)$ [8], [9].

The goal of this paper is the derivation of an $O\left(L^{2} \log L\right)$ algorithm that solves LP1 and, thus, allows the computation of the OK TFD at a cost on the same order as a fixed-kernel distribution.

This paper is organized as follows. In Section II, we introduce some notation and abstract LP1 to a slightly more general class of linear programs. In Section III, a simple algorithm based on sorting is formulated to solve a special subclass of these linear programs. Then, using this algorithm

${ }^{1}$ Since the kernel optimization is defined only in terms of the squared magnitude of the kernel, $\left|\Phi_{d}(m, n)\right|^{2}$, an additional constraint on the phase of the kernel is necessary to ensure a unique solution to the problem. Here, we assume that the phase is zero, implying that the optimal kernel is a real, nonnegative function.

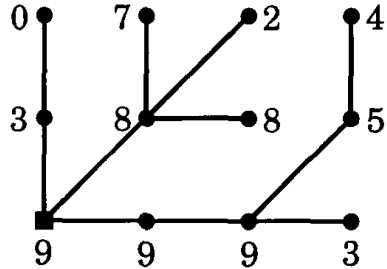

Fig. 2. A tree $\mathcal{T}$ labeled with a set of $\mathcal{T}$-nonincreasing data $C(k)$. The root node of the tree is indicated with a square. Note how the data are nonincreasing along each branch emanating from the root.

as a guide, a procedure is developed in Section IV for use in the general case. The optimality and correctness of the algorithm are proved in Section $\mathrm{V}$, while its efficient implementation is described in Section VI. A summary and discussion are presented in Section VII.

\section{PROBlem Formulation}

\section{A. Preliminaries}

We begin with some terminology that will streamline further discussion of rooted trees [10], which play a central role in subsequent sections. The nodes of a tree will be denoted by a lowercase letter, such as $k$. In a tree, there is a unique path linking any two nodes. The root of a tree will be denoted by $k=0$. For a node $k$, all nodes that lie on the path from $k$ to the root are called ancestors of $k$; all nodes that lie on paths from $k$ away from the root are called descendants of $k$. The parent of $k$ is its immediate ancestor and is denoted by $p(k)$. A node is a child of $k$ if $k$ is its parent. A node may have several children, but only one parent; nodes with no children are called leaves of the tree. We will denote a tree by the symbol $\mathcal{T}$.

Suppose that each node $k$ of a tree $\mathcal{T}$ is labeled with a real number $C(k)$. Then we can make the following definition, which is a generalization of the radially increasing constraint (4), (6) of LP1 to an arbitrary tree.

Definition 1: A function $C(k)$ defined on each node of a tree $\mathcal{T}$ is $\mathcal{T}$-nonincreasing if

$$
C(k) \leq C[p(k)] \quad \forall k \in \mathcal{T} .
$$

Functions that are not $\mathcal{T}$-nonincreasing will be referred to as $\mathcal{T}$ - nonmonotonic.

Fig. 2 illustrates a simple tree and a tree-nonincreasing function.

Define the symbol $\lfloor x\rfloor$ to be the largest integer less than or equal to the real number $x$. Similarly, define $\lceil x\rceil$ to be the smallest integer greater than or equal to $x$.

\section{B. A Generalization of $L P I$}

To generalize LP1, we replace the radial approximation tree employed in the constraint (4), (6) with an arbitrary finite tree $\mathcal{T}$ and replace $\left|A_{d}(m, n)\right|^{2}$ with an arbitrary but known set of nonnegative data $B(k)$. The variables $\left|\Phi_{d}(m, n)\right|^{2}$ are replaced with unknowns $\Psi(k)$. We will continue to refer to $\Psi(k)$ as the 
kernel of the linear program. The nodes $k$ of $\mathcal{T}$ replace the samples $(m, n)$ of the radial approximation tree. ${ }^{2}$

Making these substitutions and using Definition 1, we define the optimal kernel $\Psi_{\text {opt }}(k)$ as the optimal solution to the following linear program:

Linear Program 2 (LP2)

$$
\max _{\Psi} \sum_{k \in \mathcal{T}} B(k) \Psi(k), \quad B(k) \geq 0
$$

subject to

$$
\begin{gathered}
\Psi(k) \geq 0 \quad \forall k \\
\Psi(0)=d \\
\Psi(k) \text { is } \mathcal{T} \text { - nonincreasing } \\
\sum_{k \in \mathcal{T}} \Psi(k) \leq \gamma, \quad \gamma \geq 0 .
\end{gathered}
$$

In light of the correspondence between (5) and (12), the parameter $\gamma$ will also be referred to as the kernel volume. Without loss of generality, we can assume that the constant $d=1$. Solutions for $d \neq 1$ will simply be scaled versions of the corresponding solution for $d=1$. The number of variables in the linear program equals the number of nodes in $\mathcal{T}$ and will be denoted by $Q$.

The remainder of this paper concentrates on developing a fast, sorting-based procedure to solve LP2. The algorithm is motivated by observing that if $B$ is $\mathcal{T}$-nonincreasing then the solution to LP2 is readily obtained: simply sort the data, set $\Psi=1$ at the nodes corresponding to the $\gamma$ largest data values, and set $\Psi=0$ elsewhere. Even if $B$ is $\mathcal{T}$-nonmonotonic, nodes from sections of the tree where the data values increase can be grouped together into units called supernodes to form an equivalent set of data that is $\mathcal{T}$-nonincreasing. An identical sort and select approach can then be applied to this new data to yield the optimal kernel. As we will see, a distinctive property of the optimal kernel is that it takes on essentially only the values 1 and 0 .

\section{SOlution WITH NONINCREASING DATA}

This section describes an algorithm for solving LP2 when the data $B$ are $\mathcal{T}$-nonincreasing (see Fig. 2). Before we solve LP2, first consider the linear program obtained by relaxing the $\mathcal{T}$-nonincreasing constraint on the kernel (11) to a simple uniform boundedness constraint:

Linear Program 3 (LP3)

$$
\max _{\Psi} \sum_{k \in \mathcal{T}} B(k) \Psi(k), \quad B(k) \geq 0
$$

subject to

$$
\begin{array}{cc}
0 \leq \Psi(k) \leq 1 & \forall k \\
\sum_{k \in \mathcal{T}} \Psi(k) \leq \gamma, & \gamma \geq 0 .
\end{array}
$$

\footnotetext{
${ }^{2}$ Substituting a one-dimensional coordinate $k$ for the two-dimensional coordinates $(m, n)$ does not affect the generality of the linear program formulation.
}

This linear program is easily solved. By sorting the data in decreasing order (and renaming the nodes $0,1,2, \cdots$ according to this order)

$$
\begin{aligned}
B(0) & \geq B(1) \geq \cdots \geq B(\lfloor\gamma\rfloor) \geq \cdots \\
& \geq B(Q-1) \geq 0
\end{aligned}
$$

the optimal solution $\Psi^{\dagger}$ is obtained by starting from the left end of the list of data values and, moving right, allocating the maximum kernel volume to each node until the allowed kernel volume is exhausted

$$
\Psi^{\dagger}(k)= \begin{cases}1, & 0 \leq k<\lfloor\gamma\rfloor \\ \gamma-\lfloor\gamma\rfloor, & k=\lfloor\gamma\rfloor \\ 0, & k>\lfloor\gamma\rfloor .\end{cases}
$$

More simply, for integer $\gamma$, we set $\Psi^{\dagger}=1$ at the nodes corresponding to the $\gamma$ largest values of $B(k)$ and set $\Psi^{\dagger}=0$ elsewhere. This will be referred to as a strictly $1 / 0$ solution. For noninteger $\gamma$, we proceed in the same fashion, but set $\Psi^{\dagger}=\gamma-\lfloor\gamma\rfloor$ on node $\lfloor\gamma\rfloor$ to ensure that the volume constraint (15) remains satisfied. While the solution is no longer strictly $1 / 0$, it remains essentially $1 / 0$.

Pseudocode for this algorithm is given below. Since the procedure implicitly sorts part of the data and selects the nodes corresponding to the $\lceil\gamma\rceil$ largest data values, we have named it the sort and select algorithm (SSA). ${ }^{3}$

Sort and Select Algorithm (SSA)

Input: $B \geq 0, \gamma \geq 0$

Output: $\Psi \dagger$

Initialize:

$$
\begin{array}{ll}
\Psi^{\dagger}(k):=0 & \text { for all nodes } k \\
\Gamma:=0 & \text { (volume counter) }
\end{array}
$$

Loop:

while $\{\Gamma<\gamma\}$ do

Find:

$$
k^{*}:=\operatorname{argmax}\left\{B(k): \Psi^{\dagger}(k)=0\right\}
$$

Set:

$\Psi^{\dagger}\left(k^{*}\right):=\min (1, \gamma-\Gamma)$

$\Gamma:=\Gamma+1$

end while

end Sort and Select

Ties in the data values result in nonunique solutions to LP3 for some values of $\gamma$. For example, suppose that $\gamma$ is an integer and that the $\gamma$ th largest data value is shared by two nodes, $B(i)=B(j), i \neq j$. Then a valid solution to LP3 is obtained by setting $\Psi=1$ on nodes 0 through $\gamma-2$, setting $\Psi=\lambda, 0 \leq \lambda \leq 1$, on node $i$, and setting $\Psi=1-\lambda$ on node $j$. Nonunique solutions can be eliminated by setting up a procedure for breaking ties in data values. A simple tiebreaking procedure that can be applied whenever there is a total ordering of the nodes (as is usually the case) is to take the "larger" of two tied data values to be the one with the smaller coordinate value.

The optimality of the SSA for LP3 is easily established as a special case of Theorem 3 of Section V.

${ }^{3} \mathrm{~A}$ star (as in $k^{\star}$ ) will refer to a special node or group of nodes in the tree $\mathcal{T}$ and not the complex conjugate operation. 
Theorem I: Let $\mathcal{T}$ be a tree, $B$ be a set of arbitrary nonnegative data, and $\gamma \geq 0$. Then the kernel $\Psi^{\dagger}$ constructed by the SSA solves LP3. Furthermore, if $B$ is $\mathcal{T}$-nonincreasing, then $\Psi^{\dagger}$ also solves LP2.

Proof: The first part of the theorem follows directly from Lemma 2 of Appendix I (see Section V-A for a discussion of supernodes and the solution strategy). The second part of the theorem is established by first recognizing that if $B$ is $\mathcal{T}$. nonincreasing, then so is $\Psi^{\dagger}$. because it obeys (16) with $B$ replaced by $\Psi^{\dagger}$. Hence, $\Psi^{\dagger}$ is feasible under the constraints of both LP2 and LP3. The kernel $\Psi^{\dagger}$ also maximizes the performance measure (8), because the set of kernels satisfying the constraints (9)-(12) is a subset of the set of kernels satisfying (14) and (15).

The SSA is an example of a greedy algorithm--an algorithm that takes locally optimal steps in the hope of reaching a globally optimal solution [10]. The SSA is greedy because at each step, the contribution $B\left(k^{*}\right) \Psi\left(k^{*}\right)$ to the value of the performance measure is maximized by choosing

$$
k^{*}=\operatorname{argmax}\{B(k): \Psi(k)=0\}
$$

and setting $\Psi\left(k^{*}\right)=1$. That is, we make $\Psi$ large where $B$ is large. When the data are $\mathcal{T}$-nonincreasing, this is a globally optimal strategy for solving LP2.

\section{SOlution WITH ARBITRARY NONNEGATIVE DATA}

The SSA solves LP2 when the data $B$ are $\mathcal{T}$-nonincreasing. If this assumption is relaxed, the results can no longer be guaranteed. For example, suppose that $\gamma=1$ and the following nonmonotonic data are input to the SSA:

$$
B(k)= \begin{cases}1, & k=k^{\prime} \neq 0 \\ 0, & \text { otherwise }\end{cases}
$$

where $k^{\prime}$ is an arbitrary node different from the origin of $\mathcal{T}$. The result is a kernel $\Psi^{\dagger}=B$ which is likewise nonmonotonic and, hence, cannot be a solution to LP2.

In this section, we modify the SSA to solve LP2 with arbitrary nonnegative data. We call this improved algorithm the condensing sort and select algorithm (CSSA). Like the SSA, the CSSA is based on the greedy principle of maximizing the $B(k) \Psi(k)$ contribution to the performance measure each iteration. Unlike the SSA, the CSSA is designed to ensure that the kernel it constructs satisfies the $\mathcal{T}$-nonincreasing constraint.

\section{A. Modifying the SSA}

Consider a typical iteration of the SSA. First, we use (18) to find $k^{*}$, which has the largest data value of all nodes where currently $\Psi=0$. If $\Psi\left[p\left(k^{*}\right)\right]=1$, then we can follow the prescription of the SSA and set $\Psi\left(k^{*}\right)=1$ without violating the kernel nonincreasing constraint. The volume counter $\Gamma$ can then be incremented and control returned to the first step to select a new $k^{*}$.

However, if $\Psi\left[p\left(k^{*}\right)\right]=0$, we must proceed differently from the SSA, for setting $\Psi\left(k^{*}\right)=1$ would (at least temporarily) violate the kernel $\mathcal{T}$-nonincreasing constraint, repeated

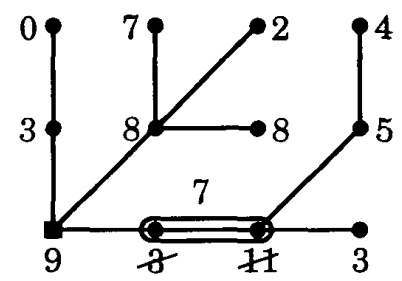

Fig. 3. Condensation of two nodes into a supernode. The data value 11 is larger than the data value 3 ; therefore the corresponding two nodes are best merged into a supernode having a supernode value (SNV) of 7 .

here for node $k^{*}$

$$
\Psi\left(k^{*}\right) \leq \Psi\left[p\left(k^{*}\right)\right] .
$$

The greedy principle suggests that since $B(k)$ is large, $\Psi\left(k^{*}\right)$ should be large as well. Unfortunately, the constraint (20) seems contrary to this goal; $\Psi\left(k^{*}\right)$ cannot be large without $\Psi\left[p\left(k^{*}\right)\right]$ being large. Maximizing $\Psi\left(k^{*}\right)$ subject to (20) yields a new compromise constraint

$$
\Psi\left(k^{*}\right)=\Psi\left[p\left(k^{*}\right)\right],
$$

which brings to light additional structure inherent in LP2, namely, that the optimal kernel must take the same value at both $k^{*}$ and $p\left(k^{*}\right)$ (this is proved in Appendix I). An important consequence of this fact is that the local contribution to the performance measure is unchanged if the data values at these two nodes are replaced by their average value, since

$$
\begin{aligned}
& B\left(k^{*}\right) \Psi\left(k^{*}\right)+B\left[p\left(k^{*}\right)\right] \Psi\left[p\left(k^{*}\right)\right] \\
& =\left(\frac{B\left(k^{*}\right)+B\left[p\left(k^{*}\right)\right]}{2}\right) 2 \Psi\left(k^{*}\right) .
\end{aligned}
$$

\section{B. Supernodes}

Properties (21) and (22) together suggest that, for the purpose of constructing the optimal kernel, nodes $k^{*}$ and $p\left(k^{*}\right)$ can be grouped or condensed into a single generalized node, or supernode. We will use uppercase script letters to denote sets of nodes; let $\mathcal{S}$ represent the supernode formed by merging nodes $k^{*}$ and $p\left(k^{*}\right)$. The average data value in a supernode is called the supernode value (SNV) and is given in this case by

$$
v(\mathcal{S})=\frac{B\left(k^{*}\right)+B\left[p\left(k^{*}\right)\right]}{2}
$$

where the function $v(\mathcal{S})$ gives the $\mathrm{SNV}$ of the supernode $\mathcal{S}$. We also write $\Psi(\mathcal{S})=\epsilon$ to indicate that $\Psi\left(k^{*}\right)=\Psi\left[p\left(k^{*}\right)\right]=\epsilon$. Fig. 3 illustrates the effect that condensing two nodes into a supernode has on the tree and data. Note how the formation of a supernode makes the data $\mathcal{T}$-nonincreasing on that part of the tree.

It is the formation of supernodes that differentiates the CSSA from the SSA and allows it to operate correctly with arbitrary data. While the concept of a supernode has been introduced using only two nodes, in general, a supernode may contain any number of nodes (in Section $V$ we give a formal definition). Fig. 4 illustrates the formation of a threenocie supernode. In general, a supernode is parameterized by the number of internal nodes it contains, which we denote by 
$n(\mathcal{S})$, its kernel value $\Psi(\mathcal{S})$ (constant over all internal nodes), and its SNV (obtained by averaging the data values of all internal nodes)

$$
v(\mathcal{S})=\frac{1}{n(\mathcal{S})} \sum_{k \in \mathcal{S}} B(k) .
$$

The contribution of a supernode $\mathcal{S}$ to the value of the performance measure is

$$
v(\mathcal{S}) n(\mathcal{S}) \Psi(\mathcal{S})
$$

(compare this with the right side of (22)). Nodes grouped into a supernode function as an indivisible unit for the remainder of the CCSA. The concepts of parent and child readily generalize to supernodes. For example, let $p(\mathcal{S})$ be the first node or supernode encountered when moving from $\mathcal{S}$ towards the root of the tree. Since a solitary node can be interpreted as a trivial supernode with $v(k)=B(k)$ and $n(k)=1$, it is convenient to assume that each node of $\mathcal{T}$ belongs to a supernode, whether trivial or otherwise. We will assume this for the remainder of the paper.

Now return to the algorithm and consider what course to take after $k^{*}$ and $p\left(k^{*}\right)$ are grouped together into a supernode $\mathcal{S}$. Note that it does not follow that we should immediately set $\Psi(\mathcal{S})=1$, for two reasons. First, it could be that $\Psi[p(\mathcal{S})]=0$, in which case setting $\Psi(\mathcal{S})=1$ would still violate the nonincreasing constraint. Second, since the SNV is always less than or equal to the data value at $k^{*}$

$$
v(\mathcal{S})=\frac{B\left(k^{*}\right)+B\left[p\left(k^{*}\right)\right]}{2} \leq B\left(k^{*}\right)
$$

there may exist another supernode $\mathcal{S}^{\prime}$ with

$$
v(\mathcal{S})<v\left(\mathcal{S}^{\prime}\right)<B\left(k^{*}\right)
$$

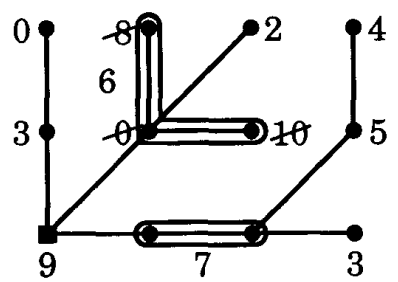

Fig. 4 Condensation of three nodes into a supernode. If the node with data value 10 is merged with the node having data value 0 , then a two-node supernode having SNV $=5$ results. However, the SNV can be increased further by merging this supernode with the node having data value 8 .

that is more appropriate for the next greedy step. Therefore, to ensure that we take a truly greedy step, we continue merging nodes into supernodes (and supernodes into larger supernodes) until we have a supernode that solves

$$
\mathcal{S}^{*}=\operatorname{argmax}\{v(\mathcal{S}): \Psi(\mathcal{S})=0\}
$$

and whose parent supernode has a kernel value of 1 .

\section{The Condensing Sort and Select Algorithm}

The last paragraph of Section IV-B defines one iteration of the CSSA: after finding $\mathcal{S}^{*}$, we either set $\Psi\left(\mathcal{S}^{*}\right)=1$ or merge $\mathcal{S}^{*}$ with $p\left(\mathcal{S}^{*}\right)$ to form a larger supernode. Iterations are continued until a kernel of volume $\gamma$ is constructed (the algorithm is shown at the bottom of this page).

The SNV and size of a supernode $\mathcal{S}$ formed by condensing two possibly nontrivial supernodes, $\mathcal{S}_{1}$ and $\mathcal{S}_{2}$, are given by

$$
v(\mathcal{S})=\frac{v\left(\mathcal{S}_{1}\right) n\left(\mathcal{S}_{1}\right)+v\left(\mathcal{S}_{2}\right) n\left(\mathcal{S}_{2}\right)}{n\left(\mathcal{S}_{1}\right)+n\left(\mathcal{S}_{2}\right)}
$$

Condensing Sort and Select Algorithm (CSSA)

Input: $B \geq 0$, tree $\mathcal{T}, \gamma \geq 0$

Output: $\Psi^{\dagger}$

Initialize nodes as trivial supernodes (do for all nodes $k$ in $\mathcal{T}$ ):

$$
\begin{aligned}
& v(k):=B(k) \\
& n(k):=1 \\
& \Psi^{\dagger}(k):=0
\end{aligned}
$$

Other initializations:

$$
\begin{array}{cl}
\Gamma:=0 & \text { (volume counter) } \\
\Psi^{\dagger}[p(0)]:=1 & \text { (it is convenient to assume that the node } p(0) \text { exists) } \\
\text { Loop: } &
\end{array}
$$$$
\text { while }\{\Gamma<\gamma\} \text { do }
$$

Find:

$$
\mathcal{S}^{*}=\operatorname{argmax}\left\{v(\mathcal{S}): \Psi^{\dagger}(\boldsymbol{S})=0\right\}
$$

if $\left\{\Psi^{\dagger}\left[p\left(\mathcal{S}^{*}\right)\right]=1\right\}$ then

Set:

$$
\begin{aligned}
& \Psi^{\dagger}\left(\mathcal{S}^{*}\right):=\min \left(1,(\gamma-\Gamma) / n\left(\mathcal{S}^{*}\right)\right) \\
& \Gamma:=\Gamma+n\left(\mathcal{S}^{*}\right)
\end{aligned}
$$

else

Condense $\mathcal{S}^{*}$ and $p\left(\mathcal{S}^{*}\right)$ into a single supernode using (29) and (30)

(keep $\Psi^{\dagger}=0$ on the new supernode)

end if

end while

end Condensing Sort and Select 


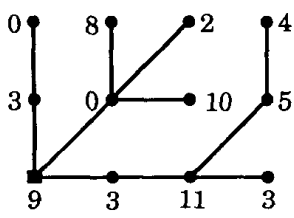

(a)

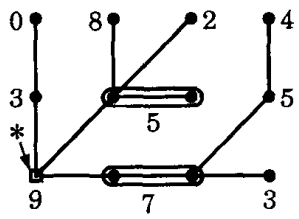

(d)

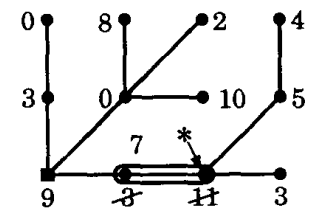

(b)

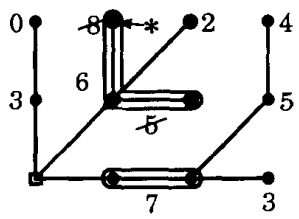

(e)

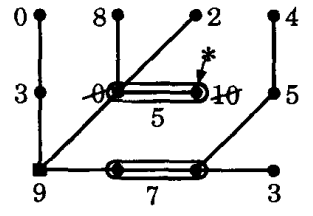

(c)

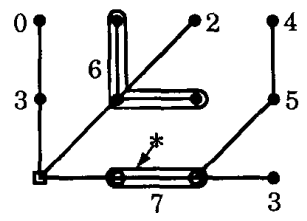

(f)

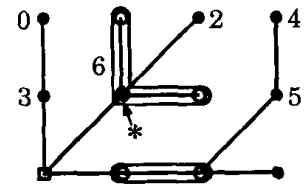

(g)

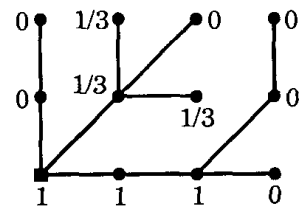

(h)

Fig. 5. Operations performed by the condensing sort and select algorithm (CSSA) to solve a linear program of the type LP2. (a) The tree $\mathcal{T}$ labeled with nonmonotonic data values $B(k)$. The desired value of kernel volume is $;=4$. (b) First iteration. The node with data value 11 is chosen as $\mathcal{S}^{*}$ (indicated by the symbol * in the figure) and merged with the node having data value 3 to form a supernode with $\mathrm{SNV}=7$. (c) Second iteration. $\mathcal{S}^{*}$ has data value 10 ; hence it is merged with its parent node to form another supernode with $\mathrm{SNV}=5$. (d) Third iteration. $\mathcal{S}^{*}$ occurs at the origin; hence $\Psi^{\dagger}$ is set to 1 there-the square denoting the root is changed to represent this. The kernel volume counter is set to $\Gamma=1$. (e) Fourth iteration. $\mathcal{S}^{*}$ has data value 8 . The corresponding node is merged with its parent supernode to form a three-node supernode having SNV=6. (f) Fifth iteration. $\mathcal{S}^{*}$ is the supernode having SNV $=7$. Since the parent supernode of $S^{*}$ is the origin and $\Psi^{\dagger}=1$ there, we set $\Psi^{\dagger}=1$ on this supernode. The kernel volume counter is now $\Gamma=3$. (g) Sixth iteration. $\mathcal{S}^{*}$ is the supernode having $\mathrm{SNV}=6$. Since $-\Gamma=1$, only a single unit of kernel volume can be distributed over this supernode before violating the kernel volume constraint. (h) The optimal kernel $\Psi^{\dagger}$ for this tree, data, and $\uparrow=4$.

$$
n(\mathcal{S})=n\left(\mathcal{S}_{1}\right)+n\left(\mathcal{S}_{2}\right) .
$$

Fig. 5 illustrates the steps taken by the CCSA to solve a simple linear problem of the type LP2.

Recall from Section III that solutions to LP3 are essentially $1 / 0$ for noninteger kernel volumes. In general, solutions to LP2 are also essentially $1 / 0$ - the optimal kernel will take the value $\delta, 0 \leq \delta<1$, on a single supernode and the values 1 and 0 on all other nodes. Essentially $1 / 0$ solutions to LP2 arise in three types of situations. As with LP3, the first type results whenever $\gamma$ is not an integer. The second type occurs when $\mathcal{L}$, the supernode selected in the final CSSA iteration, is nontrivial (that is, $n(\mathcal{L})>1$ ), and setting $\Psi(\mathcal{L})=1$ results in a kernel that violates the volume constraint (12). As Fig. 5(h) shows, this can occur even for integer $\gamma$.

The third type of essentially $1 / 0$ solution can occur whenever there exist ties in the SNV's. For example, suppose that during the final CSSA iteration two supernodes, $\mathcal{L}_{1}$ and $\mathcal{L}_{2}$. solve (28). Then selecting either $\mathcal{L}_{1}$ or $\mathcal{L}_{2}$ yields the same maximized value of the performance measure. In fact, the value is unaffected even if we set

$$
\begin{aligned}
& \Psi\left(\mathcal{L}_{1}\right)=\lambda \frac{\gamma-\Gamma}{n\left(\mathcal{L}_{1}\right)} \\
& \Psi\left(\mathcal{L}_{2}\right)=(1-\lambda) \frac{\gamma-\Gamma}{n\left(\mathcal{L}_{2}\right)}, \quad 0 \leq \lambda \leq 1 .
\end{aligned}
$$

(Here, $\Gamma$ equals the remaining kernel volume just prior to the final iteration). Not only is the solution essentially $1 / 0$-it is also nonunique. In some cases, judicious choice of $\lambda$ (either 0 or 1) may result in a strictly $1 / 0 \mathrm{kernel}$. An ordering procedure similar to that described in Section III can be applied to eliminate nonunique solutions.

\section{OPTIMALITY OF THE CSSA}

The purpose of this section is to establish the following fundamental result.

Theorem 2: Let $\mathcal{T}$ be a tree, $B$ be a set of arbitrary nonnegative data, and $\gamma \geq 0$. Then the kernel $\Psi^{\dagger}$ constructed by the CSSA solves LP2.

The CSSA forms supernodes and allocates kernel volume concurrently, complicating its analysis. Therefore, we present a three-step, indirect proof of Theorem 2 in this section. First, the notion of a supernode as introduced in Section IV is made precise. Then, using this definition, a simple solution strategy is defined and shown to solve LP2 (Theorem 3). Finally, by showing that the CSSA correctly implements the solution strategy (Theorem 4), we show that it also solves LP2.

\section{A. The Solution Strategy}

Let $\mathcal{T}_{i}$ represent the set of nodes of the tree $\mathcal{T}$ that contains node $i$ and all nodes that are descendants of node $i$. Define the root node of a connected set of nodes as the unique internal node that is an ancestor of all other internal nodes. Note that 
if $\mathcal{S}$ is a supernode rooted at node $i$, then $\mathcal{S} \subseteq \mathcal{T}_{i}$. Finally, let $k \in \mathcal{S}$ denote that node $k$ is internal to $\mathcal{S}$.

Definition 2: A connected set of nodes $\mathcal{S}$ with root node $i$ is a complete supernode if it satisfies the following three requirements $(v(\mathcal{S})$, the supernode value of $\mathcal{S}$, is defined in (24)):

1) Let $\mathcal{L}$ be any connected set of nodes rooted at node $i$ such that $\mathcal{L} \subset \mathcal{S}$. Then

$$
v(\mathcal{L}) \leq v(\mathcal{S})
$$

2) Let $\mathcal{M}$ be any connected set of nodes rooted at node $i$ such as $\mathcal{S} \subset \mathcal{M}$. Then

$$
v(\mathcal{M}) \leq v(\mathcal{S}) .
$$

3) Let $p(\mathcal{S})$ be a complete supernode that contains node $p(i)$. Then $\mathcal{S} \cap p(\mathcal{S})=\emptyset$ and

$$
v[p(\mathcal{S})] \geq v(\mathcal{S})
$$

This requirement is waived for supernodes that contain the root node $(k=0)$ of the tree $\mathcal{T}$.

Note that the third requirement is recursive in that a supernode $\mathcal{S}$ cannot be checed for completeness until all of its ancestor nodes have been formed into complete supernodes. The third requirement immediately yields the following important result.

Proposition 1: The SNV's of a set of complete supernodes are $\mathcal{T}$-nonincreasing: that is, the function $C$ defined on a set of complete supernodes as $C(k)=v(\mathcal{S})$. if $k \in \mathcal{S}$, is $\mathcal{T}$-nonincreasing.

Armed with this result, we now define a simple solution strategy that solves LP2 given arbitrary data. First, partition the tree into complete supernodes, and order and enumerate them by decreasing order of SNV

$$
\begin{aligned}
v\left(\mathcal{S}_{0}\right) & \geq v\left(\mathcal{S}_{1}\right) \geq \cdots \geq v\left(\mathcal{S}_{c}\right) \geq \cdots \\
& \geq v\left(\mathcal{S}_{P}\right) \geq 0 .
\end{aligned}
$$

Form the kernel $\Psi_{s s}$ by starting from the left end of the list and, moving right, allocating the maximum allowable kernel volume to each supernode until the total kernel volume $\gamma$ is exhausted

$$
\Psi_{s s}(\mathcal{S})=\left\{\begin{array}{ll}
1, & 0 \leq k<c \\
\delta & k=c . \\
0, & k>c .
\end{array} \quad 0 \leq \delta \leq 1\right.
$$

Note the similarity to the SSA of Section III, specifically (16) and (17). The result of the solution strategy is an essentially $1 / 0$ kernel that is feasible under the constraints (9)-(12) of LP2. In particular, the kernel is $\mathcal{T}$-nonincreasing- $\Psi_{s s}$ satisfies (35) with $v\left(\mathcal{S}_{i}\right)$ replaced by $\Psi_{s s}\left(\mathcal{S}_{i}\right)$.

\section{B. Optimality of the Solution Strategy and CSSA}

The following theorem summarizes the first half of the proof of Theorem 2. Its proof appears in Appendix I.
Theorem 3: Let $\mathcal{T}$ be a tree, $B$ be a set of arbitrary nonnegative data, and $\gamma \geq 0$. Then the kernel $\Psi_{s s}$ constructed by the solution strategy of Section V-A solves LP2.

To show that the CSSA solves LP2, it remains only to show that the CSSA creates essentially the same kernel as the solution strategy. The proof of the next theorem is given in Appendix II.

Theorem 4: Let $\mathcal{T}$ be a tree, $B$ be a set of arbitrary nonnegative data, and $\gamma \geq 0$. Then the kernel $\Psi^{\dagger}$ constructed by the CSSA is equivalent to the kernel $\Psi_{s s}$ constructed by the solution strategy of Section V-A.

The kernels constructed by the CSSA and the solution strategy are defined to be equivalent if they differ only on supernodes $\mathcal{S}_{d}$ that have $v\left(\mathcal{S}_{d}\right)=v\left(\mathcal{S}_{c}\right)$, with $\mathcal{S}_{c}$ defined as in (35) and (36). Clearly, two equivalent kernels have the same value of performance measure (8).

\section{FAST ALGORITHM IMPLEMENTATION}

The concern of this section is the efficient implementation of the CSSA. We first introduce a data structure with three distinct substructures: one each for keeping track of the branches of the tree $\mathcal{T}$, for dynamically keeping track of the supernodes, and for efficiently updating the solution to $(28) .{ }^{4}$ Then, after demonstrating how these substructures work, we consider the computational complexity of the CSSA implementation. All of the data structures utilized in this section are described in detail in $[10]$.

\section{A. Data Structure for $\mathcal{T}$}

To keep track of the static interconnections between nodes that define the tree $\mathcal{T}$ employed in the nonincreasing constraint (11), we use a data structure with three pointers. The parent pointer simply contains the value $p(k)$. In general, only parent pointers are required to completely characterize any tree. However, to increase the efficiency of the CSSA it is convenient to also have pointers to the children of each node. Two child pointers are sufficient to characterize the children of any node in $\mathcal{T}$-a FirstChild and RightSibling system is both simple and efficient [10].

\section{B. Supernode Data Structure}

Keeping track of the formation of supernodes requires a dynamic data structure for handling disjoint sets of nodes. In the course of the CSSA, the following operations are performed on supernodes:

1) Find: Given a node, $k$, determine the supernode to which it belongs.

2) Merge: Condense two supernodes (form $\mathcal{S}_{1} \cup \mathcal{S}_{2}$ ).

A simple data structure for maintaining a collection of disjoint sets that supports these two operations is an uptree structure. An up-tree structure is a collection (called a forest) of uptrees; each up-tree is a set of nodes with pointers pointing

\footnotetext{
${ }^{4} \mathrm{~A}$ word of warning: the data structures that keep track of supernodes and update the solution to $(28)$ are based on search trees [10]. Care should be taken not to confuse these trees, which merely support the implementation of the CSSA, with the tree $\mathcal{T}$ employed in the kernel nonincreasing constraint.
} 


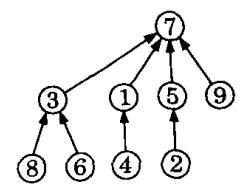

(a)
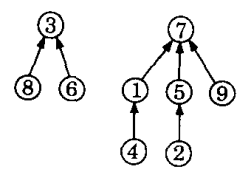

(b)
Fig. 6. Representing two supernodes with an up-tree data structure. (a) Each internal node of a supemode is associated with a node of a corresponding up-tree. The top nodes are the ones labeled $k=3$ and $k=7$. (b) To merge these two supernodes, the node $k=3$ is made to point to node $k=7$.

up the tree (see Fig. 6(a)). In our application, each up-tree corresponds to a supernode. The node at the "top" of the uptree points to itself and carries information on the status of the supernode, its SNV, number of internal nodes, kernel value, and a pointer to the root node of the supernode. Note that the top node and root node of a supernode need not be the same.

Implementation of the Find and Merge operations is simple. To find the name of, and information about, the supernode to which a node $k$ belongs, start at $k$ and follow the pointers to the top of its up-tree. (After performing this Find operation. the path from $k$ to the top node can be retraced and all nodes along the path made to point directly to the top. This path compression step increases the work done during a Find by only a constant factor, but restructures the up-tree to reduce the work required of subsequent Finds by a significant amount.) To condense two supernodes, make the top of one supernode up-tree point to the top of the other, then update the supernode information at the new top node (see Fig. 6(b)). For maximum efficiency, the up-tree with the smaller number of nodes is made to point to the up-tree with the larger number of nodes.

The up-tree data structure is very efficient. The worst-case number of computations and memory accesses required to perform a series of $n$ Merge operations is $O(n)$, while the number required to perform a series of $n$ Find operations is only $O\left(n \log ^{*} Q\right)$, where $Q$ is the total number of nodes in the uptree forest [10]. The function $\log ^{*} x$ grows sublogarithmically and is defined as

$$
\log ^{*}(x)=\text { the least } i \text { such that } \underbrace{\log _{2} \log _{2} \cdots \log _{2}}_{i} x \leq 1 .
$$

\section{Supernode Value Priority Queue}

In each iteration of the CSSA, (28) must be solved. That is, of all the supernodes where $\Psi=0$, the supernode having the largest SNV must be located. The solution is readily obtained if the top nodes of all supernodes where $\Psi=0$ are kept in a list sorted in decreasing order of SNV. Then, we simply pick the supernode corresponding to the first element on the list. However, the entries of the list change as the CSSA iterates. A supernode must be deleted from the list whenever we set $\Psi=1$ on one or merge two supernodes, while the new supernode formed by merging two supernodes must be inserted into the list at a location corresponding to its SNV. In short, the SNV list should support the following operations:

1) Find and return the supernode corresponding to the maximum SNV on the list.

2) Delete a supernode from the list.

3) Insert a new supernode into the list.
These are the specifications of a priority queue. There is a conflict between fast search and fast modification in a priority queue: fast searching requires a rigid data structure, while fast modification requires a flexible structure. There are several very efficient schemes for implementing priority queues that strike a compromise between these two extremes. Examples include balanced trees, heaps, and skip lists [10].

Balanced trees are a class of binary trees that are dynamically rearranged to allow efficient searches. Examples of balanced trees include AVL trees and 2-3 trees [10]. Their primary advantage is that for a list of $Q$ elements, each priority queue operation requires at most $O(\log Q)$ operations. The primary disadvantages of balanced trees are that they are complicated and require significant computational overhead per priority queue operation to rebalance the tree. Heaps are a second class of search trees that are simpler to implement than balanced trees, yet have equivalent worst-case performance.

Skip lists are a new data structure that combine the efficiency of balanced trees with the extreme simplicity of linked lists [11]. A skip list is a linked list with extra pointers that skip over intermediate elements of the list, allowing fast searches. The skip pointers are determined probabilistically. Advantages of skip lists include simplicity, ease of implementation, and reduced overhead per priority queue operation when combined to most balanced trees. Their main disadvantage is the lack of an $O(\log Q)$ worst-case performance bound. Rather, the performance bounds are probabilistic. For example, for a list of more than 250 elements, the probability that a search will take more than three times the expected time $(O(\log Q))$ is less than $10^{-6}$. We have utilized a skip list for our implementation of the CSSA, but we expect that a heap would offer comparable performance and ease of implementation.

\section{CSSA Data Structures in Operation}

The three distinct data structures we have discussed work together to implement the CSSA. The structure of $\mathcal{T}$ is summarized by the parent and child pointers, supernodes are represented as a forest of up-trees, and the priority queue of SNV's is kept as a (deterministically or probabilistically) balanced search tree.

Fig. 7 illustrates the block of data structure parameters that is associated with each node $k$ of $\mathcal{T}$. The parent, FirstChild, and RightSibling pointers are static parameters; the rest are dynamically updated. The root pointer is set to zero at all nodes except for top and root nodes. At a top node, the pointer holds the location of the root of the supernode. At a root node, the pointer holds a flag value (for example -1 , if all nodes are represented by positive integers) to distinguish the root node from the other nodes in a supernode. The remaining fields contain pointers that implement the SNV priority queue. The exact nature of these parameters depends on the type of queue implemented.

Referring to the CSSA pseudocode of Section IV-C, note that the main loop contains only four nontrivial operations. We now describe how these operations are implemented using the proposed data structures. 


\begin{tabular}{|c|}
\hline Node $k$ \\
\hline \hline Parent pointer \\
\hline FirstChild pointer \\
\hline RightSibling pointer \\
\hline Up-tree pointer \\
\hline Root pointer \\
\hline SNV \\
\hline Number of internal nodes \\
\hline Kernel value \\
\hline \hline SNV queue pointers \\
\hline
\end{tabular}

Fig. 7. Block of data structure parameters assigned to each node of the tree $\mathcal{T}$.

1) Find $\mathcal{S}^{*}:=\operatorname{argmax}\left\{v(\mathcal{S}): \Psi^{\dagger}(\mathcal{S})=0\right\}$.

Simply find the supernode $\mathcal{S}^{*}$ with the maximum value on the SNV priority queue. (How this is accomplished depends on the specific queue implementation.) Let $t_{1}$ denote the top node of the up-tree representing $\mathcal{S}^{*}$. Delete $t_{1}$, and hence $\mathcal{S}^{*}$, from the SNV priority queue.

2) Determine whether $\Psi^{\dagger}\left[p\left(\mathcal{S}^{*}\right)\right]=1$.

The top node of $\mathcal{S}^{*}$ is known to be $t_{1}$; find the root node of $\mathcal{S}^{*}$ using the root pointer at $t_{1}$. Call the root node $r$, and use the static parent pointer at $r$ to find $p(r)$. This is an internal node of the supernode $p\left(\mathcal{S}^{*}\right)$. Now follow the up-tree pointers from $p(r)$ to the top node (call it $t_{2}$ ) of the $p\left(\mathcal{S}^{*}\right)$ up-tree. Checking the kernel value field at $t_{2}$ completes this step. Finally, perform the path compression step, setting all up-tree pointers along the path from $p(r)$ to $t_{2}$ to point to $t_{2}$.

3) Set $\Psi^{\dagger}\left(\mathcal{S}^{*}\right):=\epsilon$.

The root node of $\mathcal{S}^{*}$ is already known from Step 2 to be $r$. To set the kernel to $\epsilon$ at all internal nodes in $\mathcal{S}^{*}$, start from $r$ and perform either a depth-first or breadth-first traversal [10] of the internal nodes of the supernode $\mathcal{S}^{*}$. These nodes form a tree (call it the supernode subtree), and the static child pointers make the traversal simple. Once all of the leaves of this subtree have been visited, the traversal is complete. A leaf of a supernode subtree is easily recognized because the root pointers all of its children are set to the flag value $(-1)$, indicating that they are root nodes of supernodes distinct from $\mathcal{S}^{*}$.

4) Condense $\mathcal{S}^{*}$ and $p\left(\mathcal{S}^{*}\right)$ into a single supernode. The top nodes of the up-trees representing $\mathcal{S}^{*}$ and $p\left(\mathcal{S}^{*}\right)$ are already known to be $t_{1}$ and $t_{2}$, respectively. Comparison of the "number of internal nodes" fields at nodes $t_{1}$ and $t_{2}$ indicates which of the up-trees is smaller. To merge the two up-trees, change the up-tree pointer of the top node of the smaller tree to point to the top node of the larger tree. The new SNV and number of nodes are calculated according to (29) and (30) and placed at the new top node. The new root of the supernode is the root of $p\left(\mathcal{S}^{*}\right)$. Finally, $p\left(\mathcal{S}^{*}\right)$ is deleted from the SNV priority queue $\left(\mathcal{S}^{*}\right.$ was deleted in Step 1), and the new supernode is inserted.

\section{E. Analysis of Computational Complexity}

It is straightforward to determine the time complexity of the CSSA implementation discussed in Section VI-D.
Theorem 5: Let $Q$ be the total number of unknowns in LP2 (the number of nodes in $\mathcal{T}$ ). Then the CSSA implemented with a balanced tree or heap SNV priority queue solves LP2 with a worst-case time complexity of $O(Q \log Q)$.

Proof: Initialization of the CSSA requires that the data values $B(k)$ be inserted into the SNV priority queue. Since there are $Q$ nodes in $\mathcal{T}$ and each Insert operation takes $O(\log Q)$ time, the total time of initialization is $O(Q \log Q)$.

Next, note that the maximum possible number of iterations of the CSSA is $Q$, because the number of supernodes where $\Psi=0$ is initially $Q$ (all nodes are initialized as trivial supernodes) and decreases by one every iteration--either because of condensing two supernodes into one supernode or because of allocating nonzero kernel volume to a supernode. The priority queue operations of Steps 1 and 4 from Section VI-D take worst-case $O(\log Q)$ time; hence the total time to complete $Q$ iterations of the CSSA is $O(Q \log Q)$. The uptree operations of Steps 2 and 4 have worst-case costs for $Q$ executions of $O\left(Q \log ^{*} Q\right)$. Therefore, the contribution of Steps 1,2 , and 4 to the total running-time of the CSSA is $O(Q \log Q)$.

For Step 3, note that each node of $\mathcal{T}$ participates in a breadth-first or depth-first traversal at most once during execution of the CSSA. Since the cost to visit a node using either traversal method is a constant, the total cost of traversals over the entire CSSA must be $O(Q)$.

Finally, all other operations take only $O(1)$ time per iteration of the CSSA. Thus, the total time-complexity of the algorithm is as stated.

An identical proof yields a probabilistic $O(Q \log Q)$ complexity bound when the SNV priority queue is implemented using a skip list.

\section{CONClusion}

In this paper, we have developed the condensing sort and select algorithm (CSSA), a procedure for solving a class of linear programs (LP2) whose characteristic feature is a tree structure that enforces a nonincreasing constraint on the unknown kernel. The time complexity of the CSSA implementation- $O(Q \log Q)$ with $Q$ representing the number of variables in the kernel-is several orders of magnitude less than the other known methods of solution [7]-[9]. This high efficiency is achieved by exploiting the simple structure of the linear program.

The structure of LP2 also leads to solutions with interesting properties. The kernel nonincreasing constraint (11) implies that the region of support of the optimal kernel both contains the origin and is connected. Furthermore, the optimal kernel is essentially $1 / 0$, with units of kernel volume allocated on supernodes, the node groupings that make the data nonincreasing along the branches of the tree.

The methods of this paper are intimately connected to the concept of dynamic programming [12]. Dynamic programming solves a complex optimization problem involving many variables by breaking it down into a sequence of smaller problems Cast into the dynamic programming mold, the CSSA (with a trivial modification allowing it to distribute only a total of one 
unit of kernel volume at a time) can be interpreted as solving a sequence of problems of the following type: Given the set of nodes where $\Psi<1$, allocate a single unit of kernel volume over these nodes in a way that maximizes the contribution of the value of the performance measure. As we have shown, the solution to this problem is to distribute the volume over the supernode corresponding to the largest SNV. Thus, the optimal kernel is "grown" out from the root of the tree in a series of stages.

An important consequence of the dynamic programming principle of optimality is that an optimal kernel is created at each step of growth; that is, the kernel of volume $\Gamma$ constructed during an intermediate iteration of the CSSA solves LP2 with $\gamma=\Gamma$. This property could prove useful in some applications. Given a fixed set of data, once the supernodes defining the optimal kernel of volume $\gamma_{1}$ are found, it is trivial to create any optimal kernel of volume $\gamma_{2}<\gamma_{1}$. Furthermore, if the optimal kernel of volume $\gamma_{3}>\gamma_{1}$ is later required, then the growth process can be continued from where it left off. In applications in which optimal kernels of several volumes are required for a fixed set of data, considerable computational savings result.

As mentioned in Section I, we are particularly interested in the linear program LP1, obtained from LP2 by substituting the AF of the signal for the data $B$ and the signal-dependent, time-frequency analysis kernel for the kernel $\Psi$. In this case, the number of variables in the kernel $Q=L^{2}$, where $L$ is the number of signal data samples to analyze; hence the computational complexity of the CSSA is on the same order as a 2-D FFT. Therefore, the total time to compute the signaldependent time-frequency distribution, including the time to compute the AF of the signal, solve LP1 for the optimal kernel, and take the 2-D Fourier transform of the AF-kernel product, is $O\left(L^{2} \log L\right)$. Since this is on the same order as a fixed-kernel time-frequency distribution, this paper has demonstrated that time-frequency analysis using a signal-dependent kernel can be competitive computationally with more limited fixed-kernel methods.

\section{APPENDIX I}

\section{PROOF OF THEOREM 3}

Theorem 3 is stated in Section V-B. Its proof follows directly from two key lemmata.

Lemma 1: Given any kernel that is feasible under the constraints (9)-(12) of LP2, there exists another kernel of the same volume that is constant on complete supernodes, bounded between 0 and 1 , and has the same or a greater value of the performance measure (8).

Proof: Let the complete supernodes be defined by the data values $B(k)$ as in the definition of Section V-A. Let $\Psi_{f}$ represent the feasible kernel, and assume that it is nonconstant on a complete supernode $\mathcal{S}$. Since $\Psi_{f}$ is $\mathcal{T}$-nonincreasing, it can be represented on $\mathrm{S}$ as a sum of constant functions defined on $n$ nested subsets of $\mathcal{S}$

$$
\mathcal{S}=\mathcal{R}_{0} \supset \mathcal{R}_{1} \supset \mathcal{R}_{2} \supset \mathcal{R}_{3} \supset \cdots \supset \mathcal{R}_{n}
$$

(see Fig. 8). That is, we decompose $\Psi_{f}$ on $\mathcal{S}$ as

$$
\Psi_{f}(k)=\sum_{i=0}^{N(k)} \epsilon_{i}, \quad k \in \mathcal{S}
$$

where $\epsilon_{i} \geq 0$ is the constant value corresponding to the set $\mathcal{R}_{i}$, and $N(k)=j$ if $k \in \mathcal{R}_{j}$ but $k \notin \mathcal{R}_{j+1}$. Note that each $\mathcal{R}_{i}$ is connected and contains the root node of $\mathcal{S}$. Therefore, from (32) of the supernode definition of Section V-A, we have

$$
v(\mathcal{S}) \geq v\left(\mathcal{R}_{i}\right) \quad \forall i .
$$

Note, also that the value of the performance measure on supernode $\mathcal{S}$ can be expressed as

$$
\begin{aligned}
\sum_{k \in \mathcal{S}} B(k) \Psi_{f}(k) & =\sum_{i=0}^{n}\left[\sum_{k \in \mathcal{R}_{i}} B(k)\right] \epsilon_{i} \\
& =\sum_{i=0}^{n} n\left(\mathcal{R}_{i}\right) v\left(\mathcal{R}_{i}\right) \epsilon_{i} .
\end{aligned}
$$

Define a new kernel $\widetilde{\Psi}$ that is constant on $\mathcal{S}$ as the average value of $\Psi_{f}$ on $\mathcal{S}$

$$
\widetilde{\Psi}(k)= \begin{cases}\frac{1}{n(\mathcal{S})} \sum_{i=0}^{n} \epsilon_{i} n\left(\mathcal{R}_{i}\right), & k \in \mathcal{S} \\ \Psi_{f}(k), & \text { otherwise. }\end{cases}
$$

Note that $\widetilde{\Psi}$ has the same volume as $\Psi_{f}$ and that $\widetilde{\Psi}$ is bounded by 0 and 1 . Computing the value of the performance measure (8) for $\widetilde{\Psi}$ and using (40)-(42) yield

$$
\begin{aligned}
\sum_{k \in \mathcal{T}} B(k) \widetilde{\Psi}(k) \\
=\sum_{k \notin \mathcal{S}} B(k) \widetilde{\Psi}(k)+\sum_{k \in \mathcal{S}} B(k)\left[\frac{1}{n(\mathcal{S})} \sum_{i=0}^{n} \epsilon_{i} n\left(\mathcal{R}_{i}\right)\right] \\
=\sum_{k \notin \mathcal{S}} B(k) \widetilde{\Psi}(k)+\left[\frac{1}{n(\mathcal{S})} \sum_{i=0}^{n} \epsilon_{i} n\left(\mathcal{R}_{i}\right)\right]\left[\sum_{k \in \mathcal{S}} B(k)\right] \\
=\sum_{k \notin \mathcal{S}} B(k) \widetilde{\Psi}(k)+\left[\frac{1}{n(\mathcal{S})} \sum_{i=0}^{n} \epsilon_{i} n\left(\mathcal{R}_{i}\right)\right] n(\mathcal{S}) v(\mathcal{S}) \\
=\sum_{k \notin \mathcal{S}} B(k) \widetilde{\Psi}(k)+\sum_{i=0}^{n} \epsilon_{i} n\left(\mathcal{R}_{i}\right) v(\mathcal{S}) \\
\geq \sum_{k \notin \mathcal{S}} B(k) \widetilde{\Psi}(k)+\sum_{i=0}^{n} \epsilon_{i} n\left(\mathcal{R}_{i}\right) v\left(\mathcal{R}_{i}\right) \\
=\sum_{k \in \mathcal{T}} B(k) \Psi_{f}(k) .
\end{aligned}
$$

Thus, the value of the performance measure does not decrease if an LP2-feasible kernel $\Psi_{f}$ is modified to be constant over a complete supernode. Performing this modification on each complete supernode where $\Psi_{f}$ is nonconstant completes the proof.

Lemma 2: Given an arbitratry set of supernodes, and assuming that the kernel must be constant on these supernodes and be bounded by 0 and 1 , a kernel of volume $\gamma$ that maximizes the value of the performance measure (8) is given by the solution strategy of Section V-A. 


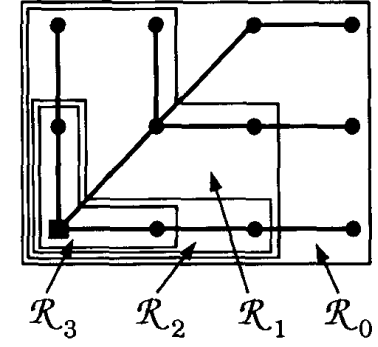

Fig. 8. Decomposition of $\mathcal{T}$-nonincreasing kernel values on a supernode into a summation of functions that are constant on the nested sets $\left\{\mathcal{R}_{i=0}^{\prime \prime}\right\}$ (here for $n=3$ ). The root node of the supernode is denoted by a square.

Proof: Let $\widetilde{\Psi}$ represent a solution to this optimization problem, and let $\Psi_{s s}$ represent the kernel constructed by the solution strategy. Note that both $\widetilde{\Psi}$ and $\Psi_{s s}$ are constant on supernodes. Define the performance measure functional $J(\Psi)=\Sigma_{k \in \mathcal{T}} B(k) \Psi(k)$ and the volume measure functional $\operatorname{vol}(\Psi)=\Sigma_{k \in \mathcal{T}} \Psi(u)$.

The optimality of $\widetilde{\Psi}$ suggests that $J(\widetilde{\Psi}) \geq J(\Psi)$ for all kernels $\Psi$ that are constant on the given set of supernodes, are bounded by 0 and 1 , and have $\operatorname{vol}(\Psi) \leq \gamma$. Assume to the contrary that the solution strategy is suboptimal; that is, assume that

$$
J\left(\Psi_{s s}\right)<J(\widetilde{\Psi})
$$

We will use this assumption to construct a kernel that contradicts the optimality of $\widetilde{\Psi}$.

This inequality has two important implications. First, the set of supernodes where $\widetilde{\Psi}$ and $\Psi_{s s}$ differ, $\mathcal{D}=\{\mathcal{S}: \widetilde{\Psi}(\mathcal{S}) \neq$ $\left.\Psi_{s s}(\mathcal{S})\right\}$. contains at least two supernodes. Certainly, for (44) to hold, we must have $\widetilde{\Psi}\left(\mathcal{S}_{a}\right)>\Psi_{s s}\left(\mathcal{S}_{a}\right)$ at some supernode $\mathcal{S}_{a}$. However, since $\operatorname{vol}\left(\Psi_{s s}\right)=\gamma$ and $\operatorname{vol}(\widetilde{\Psi}) \leq \gamma$, there must also exist some supernode $\mathcal{S}_{b}$ where $\tilde{\Psi}\left(\mathcal{S}_{b}\right)<\Psi_{s s}\left(\mathcal{S}_{b}\right)$.

Now that we know that $\mathcal{D}$ contains at least two supernodes, order the supernodes by their SNV's as in (35). Let $\mathcal{S}_{l}$ be the supernode in $\mathcal{D}$ that lies furthest to the left on the list, and let $\mathcal{S}_{r}$ be the supernode in $\mathcal{D}$ that lies furthest to the right. Immediately, we have $v\left(\mathcal{S}_{l}\right) \geq v\left(\mathcal{S}_{r}\right)$. The second consequence of (44) is that this inequality is strict

$$
v\left(\mathcal{S}_{l}\right)>v\left(\mathcal{S}_{r}\right) .
$$

Otherwise, if $v\left(\mathcal{S}_{l}\right)=v\left(\mathcal{S}_{r}\right)$, then all supernodes in $\mathcal{D}$ must have the same SNV, which in turn suggests that $J(\widetilde{\Psi})=$ $J\left(\Psi_{s s}\right)$.

The supernode $\mathcal{S}_{l} \in \mathcal{D}$; hence $\tilde{\Psi}\left(\mathcal{S}_{l}\right) \neq \Psi_{s s}\left(\mathcal{S}_{l}\right)$. In fact, we have

$$
\widetilde{\Psi}\left(\mathcal{S}_{l}\right)<\Psi_{s s}\left(\mathcal{S}_{l}\right) \leq 1
$$

To see this, assume to the contrary that $\Psi_{s s}\left(\mathcal{S}_{l}\right)<\widetilde{\Psi}\left(\mathcal{S}_{l}\right) \leq 1$. This implies that $\Psi_{s s}<1$. However, recall that $\Psi_{s s}$ results from the solution strategy and is essentially $1 / 0$; therefore if $\Psi_{s s}\left(\mathcal{S}_{l}\right)<1$, then all other supernodes in the set $\mathcal{D}$ (all of which have smaller supernode values) have $\Psi_{s s}=0$. This contradicts the existence of a supernode $\mathcal{S}_{b}$ in the set $\mathcal{D}$ with
$\Psi_{s s}\left(\mathcal{S}_{b}\right)>\widetilde{\Psi}\left(\mathcal{S}_{b}\right)$. Similar reasoning for $\mathcal{S}_{r}$, the supernode lying furthest to the right of the list, yields

$$
\widetilde{\Psi}\left(\mathcal{S}_{r}\right)>\Psi_{s s}\left(\mathcal{S}_{r}\right) \geq 0 \text {. }
$$

Let $\varepsilon=\min \left[1-\widetilde{\Psi}\left(\mathcal{S}_{l}\right), \widetilde{\Psi}\left(\mathcal{S}_{r}\right)\right]>0$, and form a new kernel $\widehat{\Psi}$ from $\widetilde{\Psi}$ by transferring some kernel volume from supernode $\mathcal{S}_{r}$ to supernode $\mathcal{S}_{l}$

$$
\widehat{\Psi}(k)= \begin{cases}\widetilde{\Psi}(k)+\frac{\epsilon}{n\left(\mathcal{S}_{l}\right)}, & k=\mathcal{S}_{l} \\ \widetilde{\Psi}(k)-\frac{\epsilon}{n\left(\mathcal{S}_{r}\right)}, & k=\mathcal{S}_{r} \\ \widetilde{\Psi}(k), & \text { otherwise. }\end{cases}
$$

Note that $\operatorname{vol}(\widehat{\Psi})=\gamma$ and that $\widehat{\Psi}$ is bounded between 0 and 1. Now, computing the value of the performance measure (8) for $\widehat{\Psi}$ and using (45) yield

$$
\begin{aligned}
J(\widehat{\Psi}) & =\sum_{k \in \mathcal{T}} B(k) \widehat{\Psi}(k) \\
& =\sum_{k \in \mathcal{T}} B(k) \widetilde{\Psi}(k)+\epsilon\left[v\left(\mathcal{S}_{l}\right)-v\left(\mathcal{S}_{r}\right)\right] \\
& >\sum_{k \in \mathcal{T}} B(k) \widetilde{\Psi}(k)=J(\widetilde{\Psi})
\end{aligned}
$$

which contradicts the optimality of $\widetilde{\Psi}$. Thus, $\widetilde{\Psi}=\Psi_{s s}$.

Proof of Theorem 3: The proof that the kernel $\Psi_{s s}$ constructed by the solution strategy solves LP2 is a straightforward application of Lemmata 1 and 2 . Let $\Psi_{\text {opt }}$ represent a solution to LP2. Application of Lemma 1 to $\Psi_{\text {opt }}$ constructs an intermediate kernel $\widetilde{\Psi}$ that is constant on complete supernodes and has the same or a greater value of the performance measure (8), that is, $J\left(\Psi_{\text {opt }}\right) \leq J(\widetilde{\Psi})$. Now, application of Lemma 2 yields $J(\widetilde{\Psi}) \leq J\left(\Psi_{s s}\right)$. since $\Psi_{s s}$ maximizes the performance measure over all kernels of the same form as $\widetilde{\Psi}$. Therefore, since

$$
J\left(\Psi_{\text {opt }}\right) \leq J(\widetilde{\Psi}) \leq J\left(\Psi_{s s}\right)
$$

and $\Psi_{s s}$ is feasible under the constraints (9)-(12) (this was discussed at the end of Section V-A), the solution strategy must solve LP2.

Proof of Theorem 1: Note that the proof of Lemma 2 does not require that the supernodes be complete. Hence, the first part of Theorem 1 can be proved by applying Lemma 2 to a set of trivial supernodes, where each supernode $\mathcal{S}_{k}$ contains only the node $k$ and $v\left(\mathcal{S}_{k}\right)=B(k)$.

\section{APPENDIX II}

\section{PROOF OF THEOREM 4}

The statement of Theorem 4 appears in Section V-B. The following simple preliminary result applies not only to supernodes, but to an arbitrary set of nodes.

Lemma 3: If a set of nodes $\mathcal{A}$ is partitioned into two nonempty, disjoint sets $\mathcal{B}$ and $\mathcal{C}$, then

$$
v(\mathcal{A}) \geq v(\mathcal{B}) \Leftrightarrow v(\mathcal{A}) \leq v(\mathcal{C}) \Leftrightarrow v(\mathcal{B}) \leq v(\mathcal{C}) .
$$

The statement remains true if the inequalities are replaced by strict inequalities. 
Proof: We prove only the forward implication of the statement; the other proofs use the same reasoning. Since $\mathcal{A}=\mathcal{B} \cup \mathcal{C}$, we can write

$$
\begin{aligned}
v(\mathcal{A}) & =v(\mathcal{B} \cup \mathcal{C}) \\
& =\frac{n(\mathcal{B})}{n(\mathcal{B})+n(\mathcal{C})} v(\mathcal{B})+\frac{n(\mathcal{C})}{n(\mathcal{B})+n(\mathcal{C})} v(\mathcal{C}) \\
& \leq \frac{n(\mathcal{B})}{n(\mathcal{B})+n(\mathcal{C})} v(\mathcal{A})+\frac{n(\mathcal{C})}{n(\mathcal{B})+n(\mathcal{C})} v(\mathcal{C})
\end{aligned}
$$

where the inequality is due to the left-hand side of $(51)$. Collecting the $v(\mathcal{A})$ terms and simplifying gives $v(\mathcal{A}) \leq$ $v(\mathcal{C})$.

Proof of Theorem 4: We now show that the CSSA as described in Section IV-C constructs a kernel equivalent to that constructed by the solution strategy and, therefore, also solves LP2. The proof involves showing that the CSSA both constructs complete supernodes according to the definition of Section V-A and selects the complete supernodes corresponding to the largest SNV's. It is important to note that the CSSA does not construct a full set of complete supernodes. Rather, it performs only enough nodal condensations to form the $c$ complete supernodes with the largest SNV's (as in (35)). For a supernode $\mathcal{S}_{i}$ to be a complete supernode, it must pass the three tests (32), (33), and (34).

Claim 1: An arbitrary (possibly incomplete) supernode formed by the CSSA always satisfies (32).

Proof: Initially, each supernode contains only a single node and, hence, trivially satisfies (32). Now consider two possibly incomplete supernodes, $\mathcal{S}$ and its parent $p(\mathcal{S})$, that each individually satisfy (32). We will show that if these two supernodes are merged by the CSSA to form a supernode $\mathcal{U}=\mathcal{S} \cup p(\mathcal{S})$, then $\mathcal{U}$ also satisfies (32).

The CSSA will not merge the two supernodes unless $v[p(\mathcal{S})] \leq v(\mathcal{S})$. Let $\mathcal{U}=\mathcal{S} \cup p(\mathcal{S})$, and note from Lemma 3 that $v[p(\mathcal{S})] \leq v(\mathcal{U}) \leq v(\mathcal{S})$. As in (32), let $\mathcal{L}$ be a set such that $\mathcal{L} \subset \mathcal{U}$ and $\mathcal{L}$ contains the root node of $\mathcal{U}$ (same as the root $p(\mathcal{S})$ ). We now show that (32) holds for $\mathcal{U}$ : that is, $v(\mathcal{L}) \leq v(\mathcal{U})$. There are three cases to consider.

If $\mathcal{L} \subset p(\mathcal{S})$ (see Fig. 9(a)), then, since $p(\mathcal{S})$ is assumed to satisfy $(32)$, we have $v(\mathcal{L}) \leq v[p(\mathcal{S})] \leq v(\mathcal{U})$.

If $\mathcal{L} \supset p(\mathcal{S})$, then $\mathcal{L}$ also contains nodes from $\mathcal{S}$ (see Fig. 9 (b)). Let $\mathcal{R}=\mathcal{L} \cap \mathcal{S}$ and note that $\mathcal{R}$ contains the root of $\mathcal{S}$, since the root is the first node of $\mathcal{S}$ that is accessible from $p(\mathcal{S})$. The set $\mathcal{S}$ is assumed to satify (32); hence $v(\mathcal{R}) \leq v(\mathcal{S})$. Therefore, using Lemma 3, we have that $v(\mathcal{L})=v[p(\mathcal{S}) \cup \mathcal{R}] \leq v[p(\mathcal{S}) \cup \mathcal{S}]=v(\mathcal{U})$

Otherwise, $\mathcal{L}$ contains some but not all nodes from both sets $p(\mathcal{S})$ and $\mathcal{S}$ (see Fig. 9(c)). Let $\mathcal{R}=\mathcal{L} \cap \mathcal{S}$ as above, and let $\mathcal{Q}=\mathcal{L} \cap p(\mathcal{S})$. Since both $\mathcal{S}$ and $p(\mathcal{S})$ satisfy (32), we have $v(\mathcal{R}) \leq v(\mathcal{S})$ and $v(\mathcal{Q}) \leq v[p(\mathcal{S})]$. Now, applying Lemma 3 twice yields $v(\mathcal{L})=v(\mathcal{Q} \cup \mathcal{R}) \leq v(\mathcal{Q} \cup \mathcal{S}) \leq v[p(\mathcal{S}) \cup \mathcal{S}]=$ $v(\mathcal{U})$. Therefore $(32)$ holds for $\mathcal{U}$ in all cases.

The above analysis applies to any intermediate supernode formed by the CSSA. Now, consider a supernode $\mathcal{S}$ that is selected by the CSSA to be allocated nonzero kernel volume. Since $\mathcal{S}$ solves (28), it has the largest SNV of all supernodes on which currently $\Psi=0$. To complete the proof that $\mathcal{S}$ is a

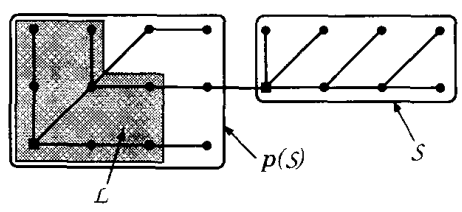

(a)

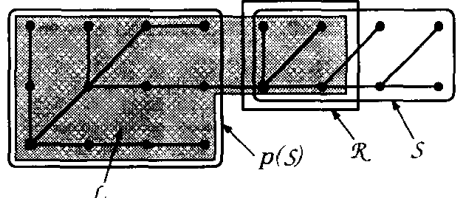

(b)

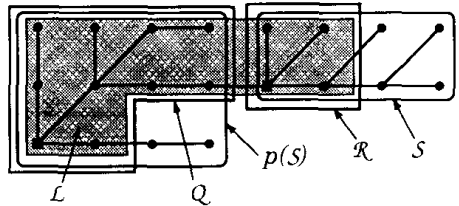

(c)

Fig. 9. A supernode $\mathcal{S}$ and its parent supernode $p(\mathcal{S})$, with (a) a subset $\mathcal{L} \subset p(\mathcal{S})$. (b) a subset $\mathcal{L} \subset[p(\mathcal{S}) \cup \mathcal{S}]$. and (c) a subset $\mathcal{L}$ that contains some but not all nodes from both $p(\mathcal{S})$ and $\mathcal{S}$. The supernodes are enclosed in boxes; the root nodes are indicated by squares. The set $\mathcal{R}$ in (b) and (c) is given by $\mathcal{R}=\mathcal{L} \cap \mathcal{S}$. The set $\mathcal{Q}$ in (c) is given by $\mathcal{Q}=\mathcal{L} \cap p(\mathcal{S})$.

complete supernode, we demonstrate that it also satisfies (33) and (34).

Claim 2: A supernode $\mathcal{S}$ allocated nonzero kernel volume by the CSSA satisfies (33).

Proof: In this case, the set $\mathcal{M}$ contains $\mathcal{S}$ and nodes in some of its (possibly incomplete) supernode descendants. For this proof only, denote the descendents of $\mathcal{S}$ as $\left\{\mathcal{S}_{i}\right\}_{i=1}^{n}$ (see Fig. 10) - do not confuse this ordering with that of (35). Decompose $\mathcal{M}$ as

$$
\mathcal{M}=\mathcal{S} \cup\left(\bigcup_{i=1}^{n} \mathcal{L}_{i}\right)
$$

where each $\mathcal{L}_{i}=\mathcal{M} \cap \mathcal{S}_{i}$. Since $M$ is connected, each $\mathcal{L}_{i}$ is connected and contains the root node of $\mathcal{S}_{i}$, because the root is the first node in $\mathcal{S}_{i}$ that is accessible from $\mathcal{S}$. By Claim 1, each $\mathcal{S}_{i}$ satisfies $(32)$; hence $v\left(\mathcal{S}_{i}\right) \geq v\left(\mathcal{L}_{i}\right)$ for all $i$. In addition, since $\mathcal{S}$ solves $(28)$, we have $v(\mathcal{S}) \geq v\left(\mathcal{S}_{i}\right) \geq v\left(\mathcal{L}_{i}\right)$ for all $i$. Therefore, since $v(\mathcal{S})$ is larger than the SNV of any node group $\mathcal{L}_{i}$ from $(55)$, it follows that $v(\mathcal{S}) \geq v\left[\mathcal{S} \cup\left(\cup_{i=1}^{n} \mathcal{L}_{i}\right)\right]=v(\mathcal{M})$. and thus (33) holds for $\mathcal{S}$.

Claim 3: A supernode allocated nonzero kernel volume by the CSSA satisfies (34) and, therefore, is complete.

Proof: The first supernode allocated nonzero kernel volume by the CSSA is $\mathcal{S}_{0}$ (see (35)). By Claims 1 and 2 and the fact that it contains the root node $(k=0), \mathcal{S}_{0}$ is complete. The next supernode allocated nonzero kernel volume, $\mathcal{S}_{1}$. satisfies (32) and (33) by Claims 1 and 2. Note, that the parent supernode of $\mathcal{S}_{1}$ is the complete supernode $\mathcal{S}_{0}$; that is, $\mathcal{S}_{0}=p\left(\mathcal{S}_{1}\right)$. Furthermore, since $\mathcal{S}_{0}$ was selected by the CSSA to receive nonzero kernel volume rather than to be merged 


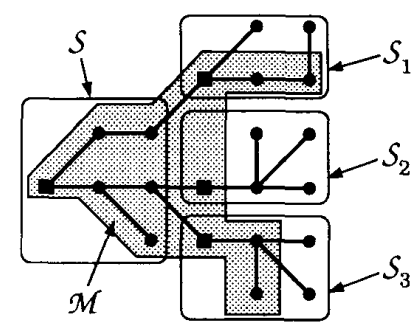

Fig. 10. A supemode $\mathcal{S}$. A set $\mathcal{M}$ such that $\mathcal{S} \subset \mathcal{M}$, and the descendam supernodes of $\mathcal{S}$ that contain nodes from $\mathcal{M}$.

with $\mathcal{S}_{1}$, we must have that $v\left(\mathcal{S}_{0}\right) \geq v\left(\mathcal{S}_{1}\right)$. Thus, $\mathcal{S}_{1}$ satisfies (34), and therefore is complete.

Continuing this process, if the first $i$ supernodes that have been allocated nonzero kernel volume by the CSSA are complete, then so is the $(i+1)$-st supernode, $\mathcal{S}_{i}$. As shown above, $\mathcal{S}_{i}$ satisfies (32) and (33). Furthermore, by the structure of the CSSA, the supernode $p\left(\mathcal{S}_{i}\right)$ must have $\Psi^{\dagger}=1$. Hence it was one of the first $i$ supernodes allocated nonzero kernel volume, and is, therefore, complete. If $\mathcal{S}_{i}$ is not complete, then it must not satisfy (34), which implies that $v\left(\mathcal{S}_{i}\right)>v\left[p\left(\mathcal{S}_{i}\right)\right]$. A contradiction arises because application of Lemma 3 to this inequality suggests that $p\left(\mathcal{S}_{i}\right)$ fails to satisfy (33) and, therefore, cannot be complete. Thus, any supernode allocated nonzero kernel volume by the CSSA must be complete.

Claim 4: The CSSA selects the $c$ supernodes having the largest SNV's.

Proof: We have shown that the CSSA constructs and selects only complete supernodes. It remains to be shown that the CSSA selects the correct complete supernodes-the $c$ supernodes having the largest SNV's. A supernode $\mathcal{S}^{*}$ cannot be selected to be allocated nonzero kernel volume unless it has the largest SNV of any set of nodes where currently $\Psi^{\dagger}=0$. This immediately implies that all supernodes selected before $\mathcal{S}^{*}$ must have had larger SNV's. Furthermore, the condensation of two supernodes $\mathcal{S}_{1}$ and $p\left(\mathcal{S}_{1}\right)$ into a larger supernode results in $v\left[\mathcal{S}_{1} \cup p\left(\mathcal{S}_{1}\right)\right], \leq v\left(\mathcal{S}_{1}\right)$ and thus $\mathcal{S}^{*}$ has a larger $\mathrm{SNV}$ than any supernode that has yet to form completely. Therefore, the CSSA forms and selects the supernodes coresponding to the largest SNV's correctly implementing the solution strategy.

\section{ACKNOWLEDGMENT}

The authors wish to thank M. Loui for suggesting the balanced-tree and up-tree data structures and for helping streamline the proofs, K. Hebel for suggesting skip lists, and J. Mast for helping with programming.

\section{REFERENCES}

[1] L. Cohen, "Generalized phase space distribution functions," J. Math Phys., vol. 7, pp. 781-786, 1966.
[2] _ "Time-frequency distributions-A review,"Proc. IEEE, vol. 77, pp. 941-981, July 1989

[3] F. Hlawatsch and G. F. Boudreaux-Bartels, "Linear and quadratic timefrequency representations," IEEE Signal Processing Mag., vol. 9, pp. 21-67, Apr. 1992

[4] H. I. Choi and W. J. Williams, "Improved time-frequency representation of multicomponent signals using exponential kernels," IEEE Trans. Acoust., Speech, Signal Processing, vol. 37, pp. 862-871, June 1989.

[5] Y. Zhao, L. E. Atlas, and R. J. Marks, "The use of cone-shaped kernels for generalized time-frequency representations of nonstationary signals," IEEE Trans. Acoust., Speech, Signal Processing, vol. 38, pp. 1084-1091, July 1990.

[6] R. G. Baraniuk and D. L. Jones, "A signal-dependent time-frequency representation: Optimal kernel design," IEEE Trans. Signal Processing, vol. 41, pp. 1589-1602, Apr. 1993.

[7] P. M. Vaidya, "Speeding up linear programming using fast matrix multiplication," in Proc. 30th Annual IEEE Symp. Found. Computer Sci., Oct. 1989, pp. 338-343.

[8] N. Meggido, "Towards a genuinely polynomial algorithm for linear programming," SIAM J. Comput., vol. 12, pp. 347-353, May 1983.

[9] B.Aspvall and Y. Shiloach, "A polynomial time algorithm for solving systems of linear inequalities with two variables per inequality," SIAM J. Comput. vol. 9, pp. 827-845, Nov. 1980.

[10] H. R. Lewis and L. Denenberg, Data Structures and Their Algorithms. New York: HarperCollins, 1991.

[11] W. Pugh, "Skip lists: A probabilistic alternative to balanced trees," Commun. ACM, vol. 33, pp. 688-676, June 1990.

[12] R. Bellman, Dynamic Programming. Princeton, NJ: Princeton University Press, 1957.

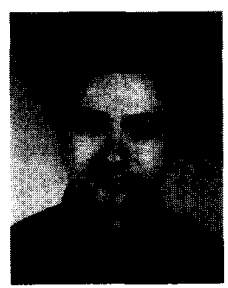

Richard G. Baraniuk (S'84-M'92) received the B.Sc. degree in 1987 from the Univeristy of Manitoba, Canada, the M.Sc. degree in 1988 from the University of Wisconsin-Madison, and the Ph.D. degree in 1992 from the Univeristy of Illinois at Urbana-Champaign, all in electrical and computer engineering.

While at the University of Illnois, he held a join appointment with the CERL Sound Group and the Coordinated Science Laboratory. During 1992-1993 he was with the Signal Processing Laboratory of Ecole Normale Supérieure de Lyon, Lyon, France, suppported by a Canadian NSERC NATO postdoctoral fellowship. Currently, he is an Assistant Professor of Electrical and Computer Engineering at Rice University in Houston, TX. His reasearch interests lie in the area of signal and image processing and include wavelet theory and time-frequency analysis.

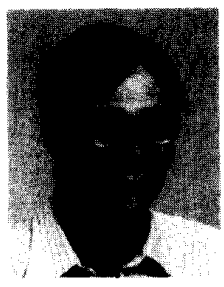

Douglas L. Jones (S'82-M'83) received the B.S.E.E. degree in 1983, the M.S.E.E degree in 1986 , and the $\mathrm{Ph} . \mathrm{D}$. degree in 1987, all from Rice University.

During the 1987-1988 academic year, he was a the University of Erlangen-Nuremberg in Germany on a Fulbright postdoctoral fellowship. He is currently an Associate Proferssor of Electrical and Computer Engineering at the University of Illinois at Urbana-Champaign, which he joined in 1988 $\mathrm{He}$ is an author of the laboratory textbook $A$ Digital Signal Processing Laboratory Using the TM32010. His research interest are in digital signal processing, including time-frequency and time-varying signal analysis, efficient algorithms for VLSI implementation, and various applications. 